University of Nebraska - Lincoln

DigitalCommons@University of Nebraska - Lincoln

\title{
The Importance of Functional Form In Optimal Control Solutions of Problems In Population Dynamics
}

\author{
Michael C. Runge \\ University of Georgia, Michael_Runge@usgs.gov \\ Fred A. Johnson \\ U.S. Fish and Wildlife Service, fred_a_johnson@fws.gov
}

Follow this and additional works at: https://digitalcommons.unl.edu/usfwspubs

Runge, Michael C. and Johnson, Fred A., "The Importance of Functional Form In Optimal Control Solutions of Problems In Population Dynamics" (2002). US Fish \& Wildlife Publications. 386.

https://digitalcommons.unl.edu/usfwspubs/386

This Article is brought to you for free and open access by the US Fish \& Wildlife Service at DigitalCommons@University of Nebraska - Lincoln. It has been accepted for inclusion in US Fish \& Wildlife Publications by an authorized administrator of DigitalCommons@University of Nebraska - Lincoln. 


\title{
THE IMPORTANCE OF FUNCTIONAL FORM IN OPTIMAL CONTROL SOLUTIONS OF PROBLEMS IN POPULATION DYNAMICS
}

\author{
Michael C. Runge ${ }^{1,3}$ And Fred A. Johnson ${ }^{2,4}$ \\ ${ }^{1}$ Warnell School of Forest Resources, University of Georgia, Athens, Georgia 30602 USA \\ ${ }^{2}$ Office of Migratory Bird Management, United States Fish and Wildlife Service, Patuxent Wildlife Research Center, \\ Laurel, Maryland 20708 USA
}

\begin{abstract}
Optimal control theory is finding increased application in both theoretical and applied ecology, and it is a central element of adaptive resource management. One of the steps in an adaptive management process is to develop alternative models of system dynamics, models that are all reasonable in light of available data, but that differ substantially in their implications for optimal control of the resource. We explored how the form of the recruitment and survival functions in a general population model for ducks affected the patterns in the optimal harvest strategy, using a combination of analytical, numerical, and simulation techniques. We compared three relationships between recruitment and population density (linear, exponential, and hyperbolic) and three relationships between survival during the nonharvest season and population density (constant, logistic, and one related to the compensatory harvest mortality hypothesis). We found that the form of the component functions had a dramatic influence on the optimal harvest strategy and the ultimate equilibrium state of the system. For instance, while it is commonly assumed that a compensatory hypothesis leads to higher optimal harvest rates than an additive hypothesis, we found this to depend on the form of the recruitment function, in part because of differences in the optimal steady-state population density. This work has strong direct consequences for those developing alternative models to describe harvested systems, but it is relevant to a larger class of problems applying optimal control at the population level. Often, different functional forms will not be statistically distinguishable in the range of the data. Nevertheless, differences between the functions outside the range of the data can have an important impact on the optimal harvest strategy. Thus, development of alternative models by identifying a single functional form, then choosing different parameter combinations from extremes on the likelihood profile may end up producing alternatives that do not differ as importantly as if different functional forms had been used. We recommend that biological knowledge be used to bracket a range of possible functional forms, and robustness of conclusions be checked over this range.
\end{abstract}

Key words: adaptive management; compensatory mortality; density-dependence; equilibrium analysis; harvest theory; optimal control theory; population dynamics; reproductive function; stochastic dynamic programming; survival function.

\section{INTRODUCTION}

Optimal control theory is finding increased application in both theoretical ecology and applied ecology (Houston et al. 1988), and is central to adaptive management, an emerging paradigm in natural resource management (Walters 1986, Lancia et al. 1996). The problem of optimal control can be stated as follows: given (1) a set of possible states for a system; (2) a set of possible actions to be taken at any point in time; (3) dynamics describing the relationship between the current state, the action taken, and the future state; and

Manuscript received 21 January 2001; revised 9 February 2001; accepted 10 June 2001; final version received 11 July 2001.

${ }^{3}$ Present address: U.S. Geological Survey, Patuxent Wildlife Research Center, 11510 American Holly Drive, Laurel, Maryland 20708 USA. E-mail: Michael_Runge@usgs.gov

${ }^{4}$ Present address: Division of Migratory Bird Management, U.S. Fish and Wildlife Service, Florida Caribbean Science Center, 7920 NW 71st Street, Gainesville, Florida 32653 USA.
(4) an objective function measuring the value of the trajectory of the system through time; find the statedependent policy of actions that optimizes the objective function (Puterman 1994). This approach has been used to analyze a wide range of questions, including life history evolution (Schaffer 1983) and the optimal timing of biological processes (Williams and Nichols 1984). One of the most fruitful areas of ecology to which this approach has been applied is optimal foraging theory (Mangel and Clark 1986, 1988, Clark and Mangel 2000), understood in a broad sense to include such problems as optimal choice of habitat patch as a function of intrinsic and extrinsic conditions (Clark and Levy 1988, Houston et al. 1988), optimal choice between mating behavior and foraging (Houston and McNamara 1986, McNamara et al. 1987), optimal size of foraging groups (Clark 1987), and optimal harvest of renewable resources by humans (Williams 1982, Walters 1986, Cohen 1987, Johnson et al. 1997). 
In this paper, we focus on the use of optimal control to affect population dynamics. While the context we describe and the language we use are relevant to management of harvested populations, the approach taken and the qualitative results should also apply to more general predator-prey problems, including problems directed at life history and behavioral evolution of predators (e.g., MacArthur 1960, Holling 1965, Slobodkin 1968). Of particular interest to us is the development of alternative models to describe system dynamics. That is, what range of functional forms for the system dynamics is admissible given the known ecology, and how do the functional form and parameterization of the system model affect the optimal policies? Using simulation methods, Pascual et al. (1997) found that model structure can have a profound effect on viability analyses-even models that fit existing data equally well could produce very different management implications. In this paper, we ask whether similar effects of model structure are found in solutions of optimal control problems.

Explicit articulation of alternative models serves to characterize "structural uncertainty," that is, what is not known about how the system behaves (Walters 1986, Nichols et al. 1995). Adaptive management, which seeks to reduce structural uncertainty while simultaneously managing the system in an optimal manner, can be expected to perform better when the alternative models used fairly describe the true uncertainty about the dynamics.

We have been involved in the application of optimal control theory to a number of harvested animal populations, including mid-continent mallards (Anas platyrhynchos, Johnson et al. 1997), eastern mallards (United States Fish and Wildlife Service 1999), northern pintail (Anas acuta, Sheaffer et al. 1999), and beaver (Castor canadensis, Runge 1999). One approach we have used to generate alternative hypotheses about system dynamics has involved positing a (single) functional form for the system dynamics (usually based on ecological principles), fitting this form to existing data, and using extreme points on confidence ellipsoids of model parameters to characterize uncertainty (e.g., Walters 1986, Johnson et al. 1997, Runge 1999). The weakness of this approach is that it depends upon the assumption of the functional form. We have come to realize that we do not have a good intuition for how functional form can affect the results that come from optimization analyses, in part because analytical solutions can rarely be found and many time-consuming numerical solutions are required to get a sense of the behavior of a model across its entire parameter space. We undertook the work herein to extend our understanding of the effect of functional form in the context of optimal control of animal populations.

As the impetus for this study arose out of our work on the management of duck harvests in the United States, it is useful to briefly describe this setting. Duck harvest regulations are established once yearly based on information about the current status of the populations and environmental conditions on the breeding grounds. Typically, the management objective is to maximize cumulative harvest over an extended time frame, possibly subject to a constraint such as a minimum goal for population size. The system dynamics are modeled with a discrete-time population model that incorporates age and sex effects. Uncertainty about the system dynamics of mallards, for example, is embodied in four alternative models (Johnson et al. 1997), formed from two hypotheses about the strength of the density dependence of recruitment (weak vs. strong), and two hypotheses about the effects of harvest on annual mortality (additive vs. compensatory). Ongoing monitoring of population size provides feedback about which of the alternative models is a better predictor of the system dynamics. While the full-fledged adaptive approach is currently only in place for midcontinent mallards, efforts are underway to develop the necessary components to manage a number of other species and stocks in this way (United States Fish and Wildlife Service 1999).

\section{Population Models}

\section{Overall structure}

We consider discrete-time population models of the following form:

$N_{t+1}=N_{t}\left(1+R\left[N_{t}\right]\right)\left(1-h_{t}\right) F\left[N_{t}\left(1+R\left[N_{t}\right]\right)\left(1-h_{t}\right)\right]$

where $N_{t}$ is the prebreeding population size at time $t$, $h_{t}$ is the harvest rate at time $t\left(0 \leq h_{t} \leq 1\right)$, and $R[\cdot]$ and $F[\cdot]$ are density-dependent functions for reproduction and survival, respectively. The sequence of events in the annual cycle of this population is (1) reproduction, (2) harvest, and (3) nonharvest mortality. Note that reproduction depends on the prebreeding density, while annual survival depends on the postharvest density. While the terminology we are using suggests a human-harvested species, such as mallard ducks, this model is applicable to other settings as well. For instance, this may be an appropriate population model for a prey species that reproduces once per cycle and faces most of its predation risk soon after young are born. In such a case, $h_{t}$ is interpreted as the rate of predation. The number of animals removed through harvest (or predation), $H$, is given by

$$
H_{t}=N_{t}\left(1+R\left[N_{t}\right]\right) h_{t} \text {. }
$$

In the absence of harvest, the equilibrium population size, $K$, is found by solving

$$
K=K(1+R[K]) F[K(1+R[K])] .
$$

If we define the survival rate at this equilibrium as $\phi$, then the solution for $K$ is as follows: 

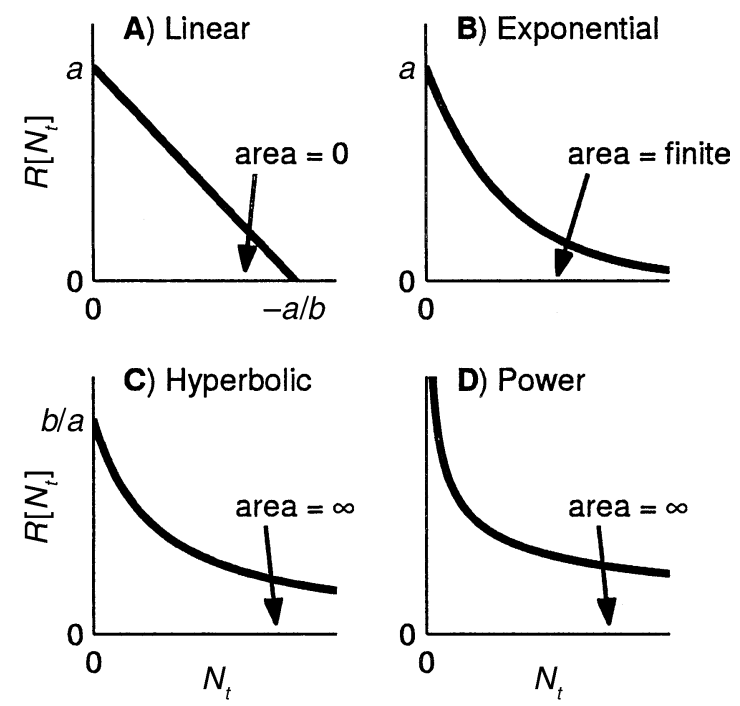

FIG. 1. Alternative models for the density-dependent reproductive function, $R\left[N_{t}\right]$. The area under the curve in the right tail of each function is shown, with an area of " 0 ", indicating that the function intercepts the $x$-axis.

$$
K=R^{-1}\left[\frac{1}{\phi}-1\right]
$$

where $R^{-1}[\cdot]$ is the functional inverse of $R[\cdot]$.

\section{Forms for the reproductive function}

In order for Eq. 4 to have a solution which represents a stable equilibrium point, $R[\cdot]$ must be a decreasing function of $N$ over some part of its range (the conditions for a stable equilibrium are actually more specific, see Yodzis 1989). For the purposes of this paper, we consider only strictly decreasing functions of $N$. Thus, we are not considering cases where there is an Allee effect, that is, where reproduction decreases at very low density (Dennis 1989). We consider three forms: linear, exponential, and hyperbolic functions (Fig. 1). A fourth function, the power function, is not tenable biologically because it allows recruitment to increase without limit at low density (Fig. 1D). The linear recruitment function is given by

$$
R\left[N_{t}\right]= \begin{cases}a+b N_{t} & \text { if } a+b N_{t}>0 \\ 0 & \text { if } a+b N_{t} \leq 0\end{cases}
$$

for $a>0$ and $b<0$. In order for the population to have $K>0, a$ must be greater than $1 / \phi-1$; otherwise, the population will decline to 0 even when not subject to harvest. The exponential recruitment function is given by

$$
R\left[N_{t}\right]=a e^{-b N_{t}}
$$

for $a>0$ and $b>0$. In order that $K>0, a$ must be greater than $1 / \phi-1$. The hyperbolic recruitment function is given by the following:

$$
R\left[N_{t}\right]=\frac{b}{a+N_{t}}
$$

for $a>0$ and $b>0$. Again, to guarantee that $K>0$, $b / a$ must be greater than $1 / \phi-1$.

One way to characterize the differences among these three recruitment functions is to consider whether the curve intercepts the $x$-axis, and if not, to consider the area in the right tail (Fig. 1). The linear function intercepts the $x$-axis (and so, in the notation of Fig. 1, is considered to have "zero" area in the tail). The exponential and hyperbolic functions are asymptotic to the $x$-axis. The area in the right tail of the exponential function is finite, because for any value $c$, the area under the curve,

$$
\int_{c}^{\infty} R\left[N_{t}\right] d N_{t}
$$

is finite. The area in the right tail of the hyperbolic function, however, is infinite.

Given the overall structure of the model, as specified in Eq. 1, there are nonetheless a very large number of forms that could be considered for the recruitment function. For biological reasons, we can eliminate all forms that are discontinuous or produce negative values over the range $0 \leq N \leq \infty$, or that are asymptotic to the $y$ axis (as we have eliminated the power function from consideration). For the purposes of this paper, let us also eliminate those that have depensatory behavior (an Allee effect). This leaves us with the class of monotonic decreasing, nonnegative functions. The simplest of this class are the three under consideration, and these three cover the three possible rates of approach to the $x$-axis. Many other forms are still possible; they will primarily differ in the number of inflection points, but all still must approach the $x$-axis at one of these three rates. The three models under consideration are closely related to widely used population models. The linear recruitment model is implicit in the discrete logistic population model. The exponential and hyperbolic recruitment functions are the Ricker (1954) and BevertonHolt (Beverton and Holt 1957) stock-recruitment functions, respectively. (Note that the most common treatment of stock-recruitment models assumes a survival rate of 0 for the breeders, but a more general stock-recruitment model allows for survival of breeders, as in Eq. 1. See, for example, Yodzis [1989]). Thus, consideration of these three models serves (1) to capture the full spectrum of possible nondepensatory recruitment models, at least to the first order of detail, and (2) to include three of the most commonly used forms.

\section{Forms for the survival function}

The survival function, $F[\cdot]$, governs the density dependence of survival outside the harvest season. This is of interest in harvest theory because it offers a mech- 

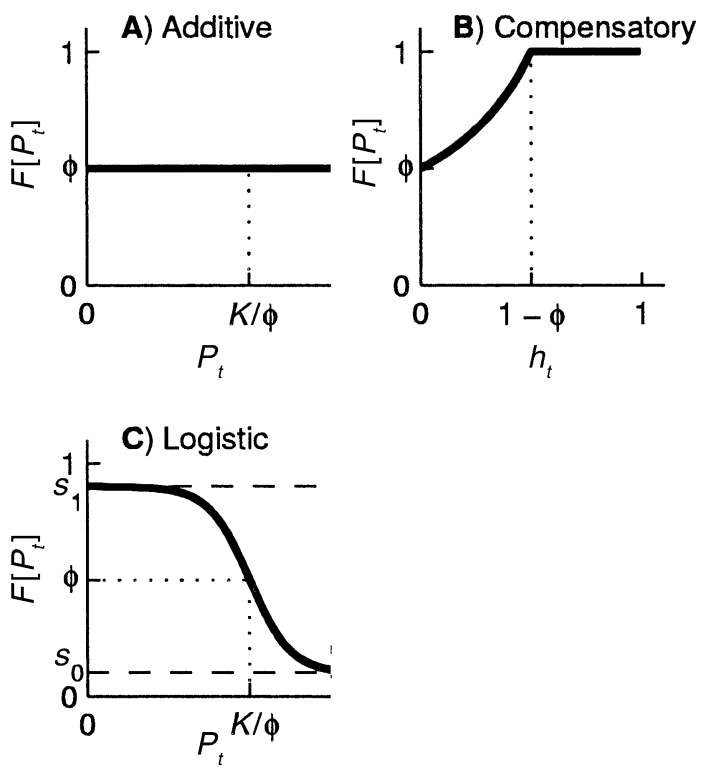

FIG. 2. Alternative models for the survival function, $F[\cdot]$. (A, C) Survival outside the harvest season is shown as a function of postharvest population size, $P_{t}$. In the absence of harvest, the survival rate is $\phi$, and the equilibrium postharvest population size is $K / \phi$. This point is shown with dotted lines. (B) Survival outside the harvest season is shown as a function of harvest rate, $h_{t}$. Harvest is compensatory up to a threshold $(1-\phi)$. In the absence of harvest, the survival rate is $\phi$.

anism for compensation: harvest mortality is offset to some degree by increases in nonharvest survival. (An important note about terminology: this use of the term "compensation" is very different from the meaning of the same term as it is applied to stock-recruitment models. In the latter application, the compensation is between increases in the population size and corresponding decreases in recruitment. In this paper, we use the first definition, in which the compensation is between harvest and nonharvest mortality.) Boyce et al. (1999) have shown that compensation for predation or harvest can arise out of density dependence in a seasonally explicit model (like the one considered here). There has been a long debate about whether harvest mortality in ducks (especially mallards) is additive to or compensatory with nonharvest mortality, with much of the debate centered on how to estimate the statistical relationship between annual survival and harvest rate (Anderson and Burnham 1976, Nichols et al. 1984, Nichols 1991, Nichols and Johnson 1996). Anderson and Burnham (1976) articulated the models governing the additive and compensatory hypotheses, the latter being, in effect, a phenomenological model that treats survival outside the harvest season as a function of harvest rate. Recent work has begun to look at a more mechanistic model, treating survival outside the harvest season as a function of population density (Johnson et al. 1993). We consider all of these possibilities in this paper (Fig. 2).
In the model under consideration (Eq. 1), survival outside the harvest season, $F[\cdot]$, is a function of the postharvest population size. Let the postharvest population size be given by

$$
P_{t} \equiv N_{t}\left(1+R\left[N_{t}\right]\right)\left(1-h_{t}\right)
$$

When there is no harvest and the population is at its equilibrium value, $K$, the postharvest population size is

$$
P_{t}=K\left(\frac{1}{\phi}\right)(1-0)=\frac{K}{\phi} .
$$

The annual survival rate in this circumstance is $\phi$ (by definition). Thus, a constraint on the function $F[\cdot]$ is that

$$
F\left[\frac{K}{\phi}\right]=\phi
$$

In the additive model, $F[\cdot]$ is not affected by the harvest rate or the population density. That is, the additive model (Fig. 2A) is density independent, and is given by

$$
F\left[P_{t}\right]=\phi .
$$

In the phenomenological compensatory model (hereinafter referred to as the "compensatory model"), survival outside the harvest season depends on harvest rate, but not on population density. For harvest rates below a certain threshold $c(c \leq 1-\phi)$, the total annual survival rate (i.e., $\left.\left(1-h_{t}\right) F[\cdot]\right)$ is $\phi$; above this threshold, total annual survival is $1-h_{t}$. Thus, harvest below the threshold rate has no impact on the population. Herein, we take the threshold to be its maximum possible value $(c=1-\phi)$ to make the difference between the additive and compensatory models most pronounced (the qualitative results shown do not depend on this choice of $c$ ). Written in the terminology of Eq. 1 , for the compensatory model (Fig. 2B),

$$
F\left[h_{t}\right]= \begin{cases}\frac{\phi}{1-h_{t}} & \text { if } h_{t} \leq 1-\phi \\ 1 & \text { if } h_{t}>1-\phi .\end{cases}
$$

We also consider a mechanistic model for density-dependent survival, the logistic model (Fig. 2C). The maximum survival rate outside the harvest season is $s_{1}$, so $F[0]=s_{1}$; and the minimum is $s_{0}$, so

$$
\lim _{P \rightarrow \infty} F\left[P_{t}\right]=s_{0} \text {. }
$$

It is implicitly assumed that $s_{0}<\phi<s_{1}$. The logistic survival model (Fig. 2C) is given by

$$
F\left[P_{t}\right]=s_{0}+\left(s_{1}-s_{0}\right)\left(\frac{1+e^{-m c}}{1+e^{m\left(P_{t}-c\right)}}\right)
$$

with the constraints

$$
\begin{aligned}
& m>\frac{\phi}{K} \ln \left(\frac{2\left(s_{1}-\phi\right)}{\phi-s_{0}}+1\right) \\
& c=\frac{1}{m} \ln \left\{\frac{\phi-s_{0}}{s_{1}-\phi}\left(e^{(m k / \phi)}-1\right)-1\right\}
\end{aligned}
$$


needed to guarantee that $c>0$ and $F[K / \phi]=\phi$. In the logistic model, $c$ is the inflection point on the curve $F[\cdot]$. It is not strictly necessary that $c>0$. If $c<0$, the curve shows exponential decline with no inflection point over the biologically relevant range for $P_{t}$.

There are a large number of functional forms that could be considered for the survival function in Eq. 1 . The biological constraints are that the function be bounded on the closed interval $[0,1]$, and continuous for positive values of $P_{t}$ (perhaps only up to some large maximum value). We have chosen to consider functions (additive, logistic) that are nonincreasing for positive $P_{p}$, but functions that are increasing over some interval are possible, for example, if there is a group effect on survival (as suggested by some of the hypotheses for brood amalgamation [Eadie et al. 1988]). The additive model is the only possible density-independent survival model. However, there are many possible nonincreasing density-dependent survival models. The logistic model used here (Eq. 15) is a flexible and intuitive one. It is the underlying model for logistic regression, a commonly used tool for survival analysis (McCullagh and Nelder 1989), and Johnson et al. (1993) suggest its use in the context of duck population models. As noted above, the compensatory model (Eq. 13) posits a very different mechanism for changes in survival rate, making them dependent on harvest rate, not population size. This model cannot be eliminated on biological grounds, although many duck biologists consider it an unlikely mechanism (Nichols 1991, Johnson et al. 1993). We included it here for three reasons: (1) it offers a very different mechanism for changes in nonharvest survival, which supports our goal of exploring effects of model structure; (2) it has a rich history in the duck literature; and (3) perhaps most importantly, it is currently one of the alternative models being used to set duck harvest regulations in the United States (United States Fish and Wildlife Service 1999). In sum, our choice of these three survival models serves to capture the three primary hypotheses about effects on nonharvest mortality.

\section{The Optimal Control Problem and the EQUILIBRIUM SOLUTIONS}

\section{The optimal control problem}

Given the state of the system, $N_{t}$, what decision (i.e., harvest rate $h_{t}$ ) is optimal with regard to the long-term objective of maximizing harvest? Let $h_{t}=D\left[N_{t}\right]$ be a decision strategy that specifies harvest rate as a function of population size. The state dynamics are given by Eq. 1. The harvest achieved in a particular year is given by Eq. 2. The value, $V[\cdot]$, of a particular decision strategy, $D[\cdot]$, is the sum of harvests over the time horizon $t=0,1, \ldots T$ achieved by using that strategy to determine harvest rate as follows:

$$
\begin{aligned}
V\left(D\left[N_{t}\right]\right) & =\sum_{t=0}^{T}\left\{H_{t} \mid h_{t}=D\left[N_{t}\right]\right\} \\
& =\sum_{t=0}^{T} N_{t}\left(1+R\left[N_{t}\right]\right) D\left[N_{t}\right] .
\end{aligned}
$$

The optimal decision strategy, $D^{*}\left[N_{t}\right]$, satisfies

$$
V^{*}\left(D^{*}\left[N_{t}\right]\right)=\max _{D[\cdot]} V\left(D\left[N_{t}\right]\right)
$$

If we consider the infinite time horizon $(T=\infty)$, then what we seek is the time-independent decision strategy, $D^{*}[N]$, that specifies state-dependent harvest rates to maximize the accumulated harvest.

The dynamics of this control process over time will exhibit the following behavior. Starting from a given initial state $\left(N_{0}=n_{0}\right)$, the population size, $N_{t}$, and harvest rate, $h_{t}=D^{*}\left[N_{t}\right]$, will converge toward an equilibrium point. Once the equilibrium point is reached, the population size and harvest rate will remain constant. The equilibrium point will be the harvest rate and associated stable population size that provide the maximum annual harvest. In other words, to maximize total harvest over an infinite time horizon, the best strategy is to efficiently reach an equilibrium point that provides the maximum sustainable annual harvest. We first consider the properties of the equilibrium point, then turn our attention to the properties of the optimal path leading to the equilibrium point.

\section{Finding the equilibrium solution}

The problem of finding the equilibrium solution can be restated in the following manner. Suppose that you choose a harvest rate and apply it without regard to the state of the system, that is, $D\left[N_{t}\right]=h_{t}=h$. Under certain conditions on $R[\cdot], F[\cdot]$, and $h$, the population size will converge to a stable value, $N_{\text {eq }}$, such that

$$
\begin{aligned}
N_{\mathrm{eq}}(h)= & N_{\mathrm{eq}}\left(1+R\left[N_{\mathrm{eq}}\right]\right)(1-h) \\
& \times F\left[N_{\mathrm{eq}}\left(1+R\left[N_{\mathrm{eq}}\right]\right)(1-h)\right]
\end{aligned}
$$

where it is understood that $N_{\text {eq }}$ is a function of $h$ (if $h$ $\left.=0, N_{\mathrm{eq}}=K\right)$. The annual harvest achieved is

$$
H(h)=N_{\mathrm{eq}}\left(1+R\left[N_{\mathrm{eq}}\right]\right) h .
$$

The optimal harvest rate, $h^{*}$, then, is the value of $h$ that maximizes $H(h)$, and can be found by solving

$$
\left.\frac{d H}{d h}\right|_{h=h^{*}}=0 .
$$

After solving for $h^{*}$, one must ascertain that $H^{\prime \prime}\left(h^{*}\right)<$ 0 , to be sure this is a maximum point. The optimal equilibrium population size is $N^{*}=N_{\mathrm{eq}}\left(h^{*}\right)$ and the optimal annual harvest is $H^{*}=H\left(h^{*}\right)$.

These equations can be solved in closed form for six out of the nine combinations of recruitment and survival functions, namely, for the cases when the survival model is either additive (Eq. 12) or compensatory (Eq. 
13). For the additive survival model, Eq. 20 can be written as

$$
1=\left(1+R\left[N_{\mathrm{eq}}\right]\right)(1-h) \phi
$$

the solution for which is

$$
N_{\text {eq }}=R^{-1}\left[\frac{1-\phi(1-h)}{\phi(1-h)}\right] \text {. }
$$

The annual harvest at equilibrium is

$$
H(h)=\frac{N_{\mathrm{eq}}}{\phi}\left(\frac{h}{1-h}\right)
$$

and the optimal harvest rate is found by taking the derivative of Eq. 25, setting it equal to 0, and solving for $h$. A detailed demonstration of these closed form solutions is shown in the Appendix.

For the compensatory model, Eq. 20 can be rearranged to

$$
R\left(N_{\mathrm{eq}}\right)= \begin{cases}\frac{1-\phi}{\phi} & \text { if } h \leq 1-\phi \\ \frac{h}{1-h} & \text { if } h>1-\phi .\end{cases}
$$

The annual harvest at equilibrium is

$$
H(h)= \begin{cases}N_{\mathrm{eq}}\left(\frac{h}{\phi}\right) & \text { if } h \leq 1-\phi \\ N_{\mathrm{eq}}\left(\frac{h}{1-h}\right) & \text { if } h>1-\phi\end{cases}
$$

and the optimal harvest rate is found by taking the derivative of Eq. 27, setting it equal to 0, and solving for $h$.

For the logistic survival functions, closed form solutions for the optimal equilibrium cannot be found (using the recruitment functions considered here). Instead, the solutions must be found numerically, after all parameters are specified. The equilibrium population size as a function of harvest rate is found by solving Eq. 20 numerically (e.g., top panel in Fig. 3) for a range of harvest rates. These results are then used to calculate the annual harvest at equilibrium (e.g., bottom panel in Fig. 3). The maximum value of $H(h)$, and the corresponding optimal harvest rate, $h^{*}$, can then be found by inspection.

We derived the closed form solutions for six model combinations, the additive or compensatory survival model combined with each of the three recruitment models. The optimal harvest rate $\left(h^{*}\right)$, optimal equilibrium population size $\left(N^{*}\right)$, and optimal annual harvest $\left(H^{*}\right)$ for these six model combinations are shown in Tables 1 and 2. In addition, we calculated numerical solutions for 18 parameterizations of the nine models considered in this paper (Table 3 ). For all models, we used the same equilibrium survival rate in the absence of harvest $(\phi=0.7)$ and chose parameters that gave
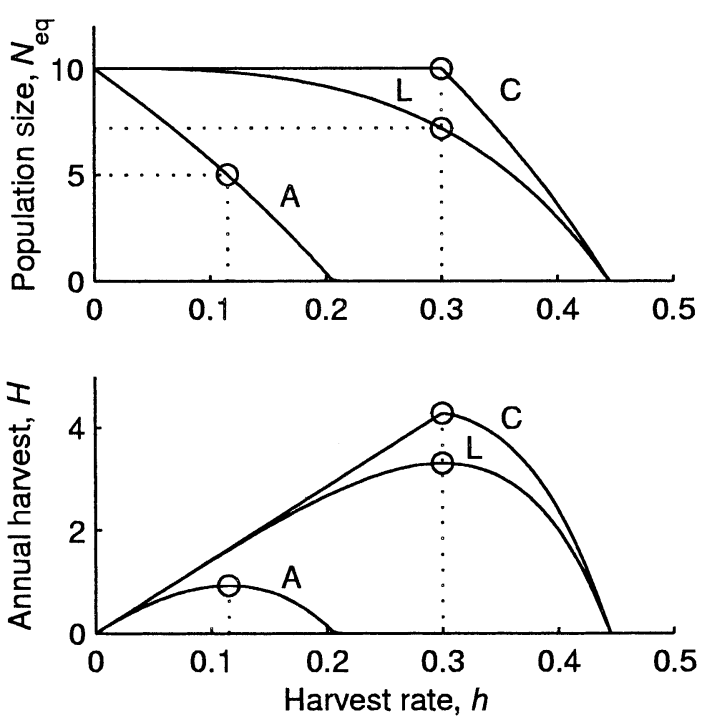

FIG. 3. Equilibrium curves under linear recruitment, model 1. The three curves in each graph refer to the additive (A), compensatory (C), and logistic (L) survival functions. The optimal equilibrium solution is indicated for each curve with dotted lines and an open circle. (Top panel) Equilibrium population size as a function of a fixed harvest rate; (bottom panel) corresponding annual harvest as a function of fixed harvest rate. The open circles indicate the maximum sustainable yield. See Table 3 for the parameter values used for this recruitment model.

the same equilibrium population size in the absence of harvest $(K=10)$. For each recruitment model (linear, exponential, hyperbolic), we chose two parameterizations representing extremes of behavior. The linear and exponential recruitment functions have different solutions under the compensatory model, depending on the maximum recruitment value (see Table 2 ). So we chose one parameterization in each range. We chose two parameterizations of the hyperbolic recruitment model that differed in their maximum recruitment (at $N=0$ ) by an order of magnitude. The parameter values used for these six recruitment models are shown in the first column of Table 3. Given the survival in the absence of harvest, $\phi$, there is only one possible parameterization of the additive survival model, and one of the compensatory model. For the logistic survival model, with $s_{0}=0$ and $s_{1}=1$, the parameter $m$ must be greater than 0.043 to insure that the inflection point occurs at $P_{t}>0$ (Eq. 16). We used $m=0.25$, which gives a moderately quick decline in survival with increase in postharvest population size. The second constraint (Eq. 17) then requires that $c=17.511$. The parameterizations of the survival models depend only on $\phi$, not on the parameterization or form of the recruitment model. We calculated numerical solutions for the optimal harvest rate $\left(h^{*}\right)$, optimal equilibrium population size $\left(N^{*}\right)$, and optimal annual harvest $\left(H^{*}\right)$ for these 18 model combinations (Table 3 ). Representative 
TABLE 1. Closed form equilibrium solutions under the additive survival model.

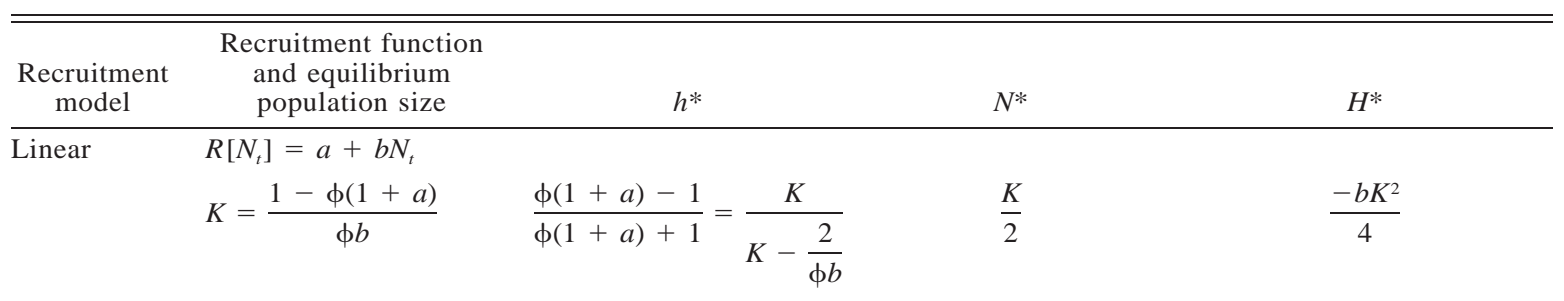

Exponential $\dagger \quad R\left[N_{t}\right]=a e^{-b N_{t}}$

$$
K=-\frac{1}{b} \ln \left(\frac{1-\phi}{\phi a}\right)
$$$$
\frac{1-\phi}{1-W\left[\frac{1-\phi}{a \phi} e^{1}\right]}-\phi
$$

$$
K+\frac{1}{b} \ln W\left[\frac{1-\phi}{a \phi} e^{1}\right] \quad N^{*}\left(\frac{1}{W\left[\frac{1-\phi}{a \phi} e^{1}\right]}-1\right)\left(\frac{1-\phi}{\phi}\right)
$$

Hyperbolic $\quad R\left[N_{t}\right]=\frac{b}{a+N_{t}}$

$$
K=\frac{b \phi}{1-\phi}-a
$$

$$
1-\frac{1}{\sqrt{\frac{b \phi(1-\phi)}{a}}+\phi}
$$

$$
\sqrt{a(K+a)}-a
$$$$
N^{*}\left(\frac{h^{*}}{\phi\left(1-h^{*}\right)}\right)
$$

$\dagger W[\cdot]$ is the Lambert $W$-function (Corless et al. 1996), which has the property $\ln W[x]+W[x]=\ln x$. While it needs to be evaluated numerically, at least one widely distributed mathematics package (Maple) has a built-in routine to do this.

graphs of these numerical solutions, for two model combinations, are shown in Figs. 3 and 4.

\section{The equilibrium solutions}

The closed form optimal equilibrium solutions under the additive and compensatory survival models (Tables 1 and 2) reveal a number of results. First, note that the results for the additive survival/linear recruitment model are the well-known results for a discrete logistic population model subject to harvest: the maximum sustained yield occurs when the population is held at half the carrying capacity. Second, under the compensatory survival model, harvest rates below $1-\phi$ have no impact on the population, and so there is always a portion of harvest that is "free." Harvesting at this threshold harvest rate holds the population at $K$. Under

TABLE 2. Closed form equilibrium solutions under the compensatory survival model.

\begin{tabular}{lcccc}
\hline \hline $\begin{array}{c}\text { Recruitment } \\
\text { model }\end{array}$ & Condition & $h^{*}$ & $N^{*}$ & $H^{*}$ \\
\hline Linear & $a<\frac{2(1-\phi)}{\phi}$ & $1-\phi$ & $K$ & $K\left(\frac{1-\phi}{\phi}\right)$ \\
& Otherwise & $\frac{a}{a+2}$ & $-\frac{a}{2 b}$ & $-\frac{a^{2}}{4 b}$ \\
Exponential & $a<\frac{e(1-\phi)}{\phi}$ & $1-\phi$ & $K$ & $K\left(\frac{1-\phi}{\phi}\right)$ \\
& Otherwise & $\frac{a}{a+e}$ & $\frac{1}{b}$ & $\frac{a}{b e}$ \\
Hyperbolic & Always & $1-\phi$ & $K$ & $K\left(\frac{1-\phi}{\phi}\right)$ \\
\hline
\end{tabular}

the linear and exponential recruitment models, if the maximum recruitment $(a)$ is below some value, the optimal solution under the compensatory survival model is to harvest at this threshold $(1-\phi)$; but if $a$ is above a certain value, the optimal harvest rate is above the threshold harvest rate and the population is held at some level lower than $K$. In the hyperbolic recruitment model, however, the optimal solution is always at the threshold harvest rate, regardless of the values of the parameters in the recruitment model. Third, for a given recruitment model, we can compare the optimal harvest rates under the additive and compensatory survival models. Under the linear and exponential recruitment models, the optimal harvest rate for the additive model is always less than that for the compensatory model. However, for the hyperbolic recruitment model, parameterizations can be found where the optimal harvest rate under the additive model is higher than under the compensatory model. Specifically, this will occur when

$$
\frac{b}{a}>\frac{\left(\frac{1}{\phi}-\phi\right)^{2}}{\phi(1-\phi)} \text {. }
$$

Fourth, the optimum equilibrium population size $\left(N^{*}\right)$ and the maximum annual harvest $\left(H^{*}\right)$ are always greater under the compensatory survival model than under the additive survival model, no matter what recruitment function is used. A graphical comparison of two extremes of behavior (Figs. 3 and 4) sheds some light on these phenomena. When the optimal harvest rate under the additive model is greater than under the compensatory model (Fig. 4), the optimal population size is much lower for the additive model. Holding the 
TABLE 3. Optimal equilibrium solutions (harvest rate, $h^{*}$; population size, $N^{*}$; and annual harvest, $H^{*}$ ) for 18 scenarios generated from six recruitment models and three survival models.

\begin{tabular}{llccc}
\hline \hline \multirow{2}{*}{$\begin{array}{c}\text { Recruitment } \\
\text { model } \dagger\end{array}$} & & \multicolumn{3}{c}{ Survival model $\neq$} \\
\cline { 2 - 5 } & Solution & Additive $\$$ & Compensatory & Logistic II \\
\hline 1) Linear & $h^{*}$ & 0.12 & 0.30 & 0.30 \\
$a=0.80$ & $N^{*}$ & 5.00 & 10.00 & 7.19 \\
$b=-0.0371$ & $H^{*}$ & 0.93 & 4.29 & 3.31 \\
2) Linear & $h^{*}$ & 0.36 & 0.50 & 0.50 \\
$a=2.00$ & $N^{*}$ & 5.00 & 6.36 & 5.91 \\
$b=-0.1571$ & $H^{*}$ & 3.93 & 6.36 & 6.06 \\
3) Exponential & $h^{*}$ & 0.15 & 0.30 & 0.32 \\
$a=1.0$ & $N^{*}$ & 4.44 & 10.00 & 6.89 \\
$b=0.0847$ & $H^{*}$ & 1.15 & 4.29 & 3.39 \\
4) Exponential & $h^{*}$ & 0.32 & 0.42 & 0.44 \\
$a=2.0$ & $N^{*}$ & 3.94 & 6.49 & 5.55 \\
$b=0.1540$ & $H^{*}$ & 2.61 & 4.78 & 0.34 \\
5) Hyperbolic & $h^{*}$ & 0.26 & 0.30 & 6.37 \\
$a=2.7273$ & $N^{*}$ & 3.16 & 10.00 & 3.48 \\
$b=5.4545$ & $H^{*}$ & 1.57 & 4.29 & 0.50 \\
6) Hyperbolic & $h^{*}$ & 0.64 & 0.30 & 3.92 \\
$a=0.2190$ & $N^{*}$ & 1.28 & 10.00 & 4.06 \\
$b=4.3796$ & $H^{*}$ & 3.19 & 4.29 & \\
\hline
\end{tabular}

$\dagger$ In all cases, the annual survival rate at equilibrium without harvest, $\phi$, was 0.7 . The parameters for each model were chosen to give $K=10.00$ (see formulas for $K$ in Table 1).

$\ddagger$ For the logistic model, $m=0.25, c=17.511$, and $s_{0}$ and $s_{1}$ were set at 0 and 1 , respectively.

$\S$ Solutions for the additive model were found by using the formulas shown in Table 1.

$\|$ Solutions for the compensatory model were found by using the formulas shown in Table 2.

II Solutions for the logistic model were found numerically.

population at such a low level results in much greater recruitment and allows a higher harvest rate.

Numerical solutions for the cases involving the logistic survival model shed light on its properties. First, the density-dependent logistic survival model has optimal equilibrium population size and maximum annual harvest intermediate between those in the additive and compensatory models. Thus, the logistic survival mod-
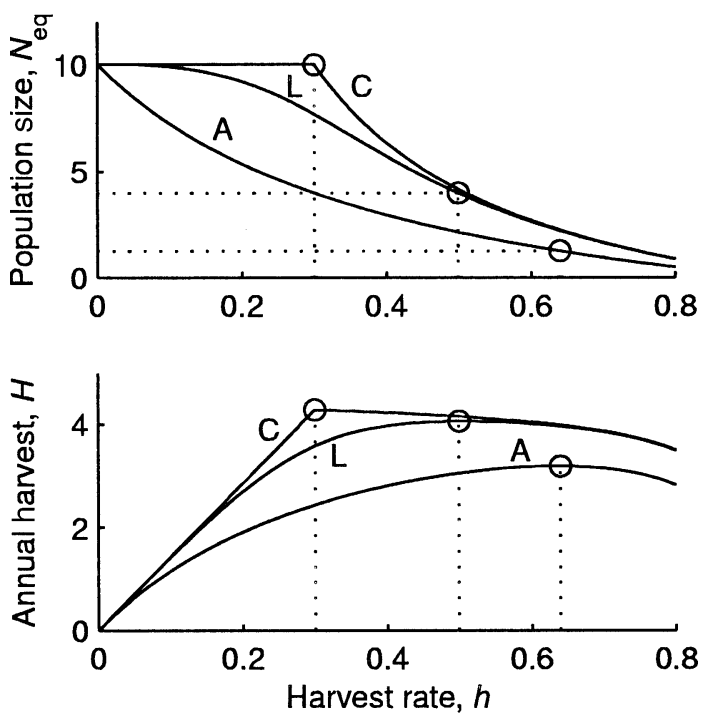

FIG. 4. Equilibrium curves under hyperbolic recruitment, model 6. See Fig. 3 for description. el does exhibit the property of compensation because increased harvest reduces the equilibrium population size, but this results in a higher survival rate outside the harvest season. Second, the optimal harvest rates for the logistic model are not necessarily intermediate between those of the additive and compensatory models (e.g., recruitment model 5 in Table 3). Third, there are cases when the optimal harvest rate under the additive survival model is greater than under the logistic model, although apparently only for the hyperbolic recruitment model (e.g., recruitment model 6 in Table 3, Fig. 4). These last two points can be explained by noting that total annual harvest, which is to be maximized, is a product of preharvest population size and harvest rate. To understand the optimal harvest rate, it is important to note the optimal equilibrium population size and the recruitment it produces. For instance, in model 6 , recruitment increases sharply as the population size decreases. Thus, by holding the population at a low size, there is tremendous recruitment, much of which can be harvested. Thus, the harvest rate can be greater than in the logistic model, even though the total harvest is less.

\section{The Optimal Control Solution AWAY FROM EQUILIBRIUM}

\section{Methods}

The preceding results describe the properties of the equilibrium solution to the optimal control problem we considered. We were also interested in how the func- 

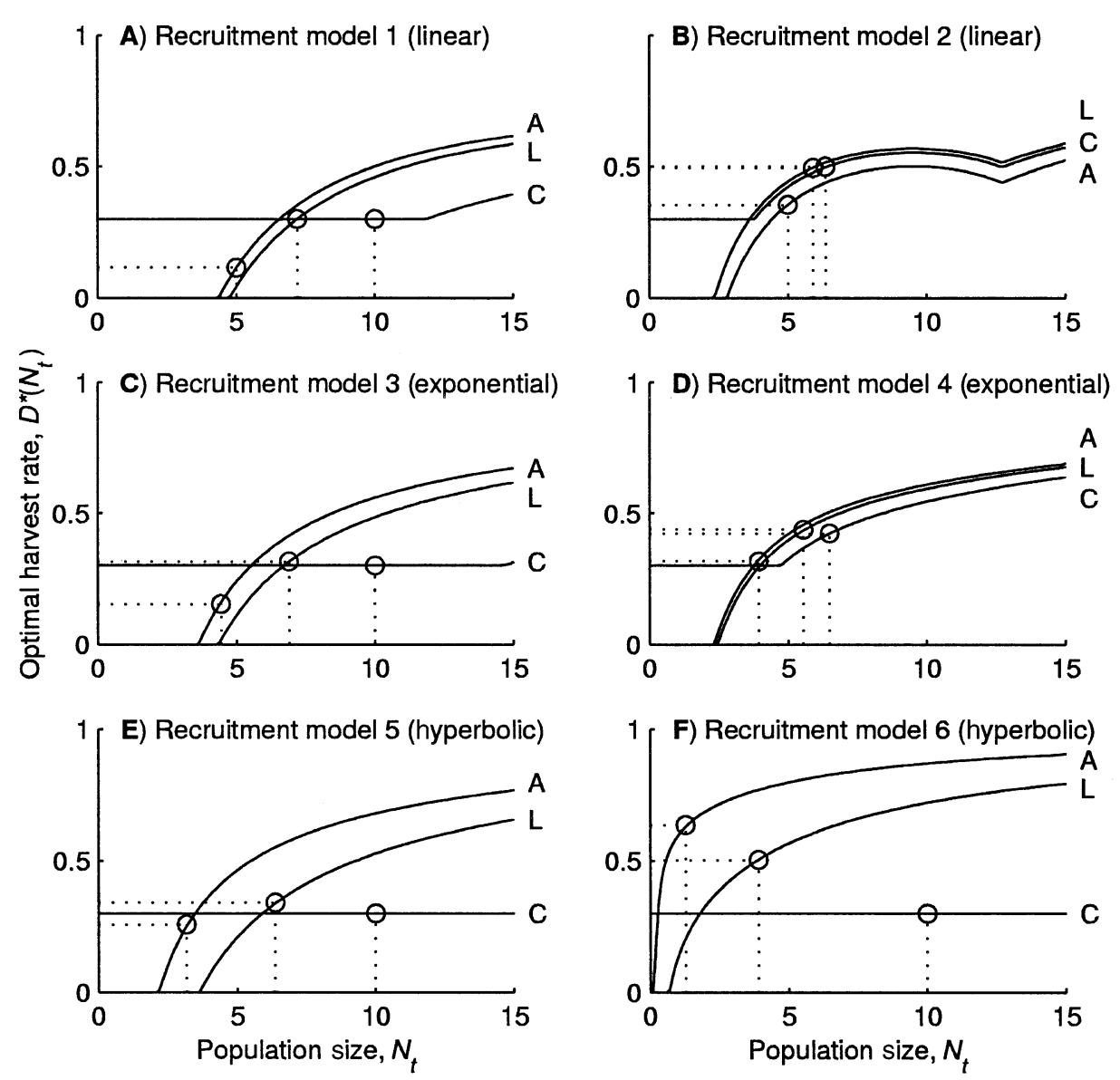

FIG. 5. State-dependent optimal harvest rate as a function of current population size, deterministic results. The three curves in each graph refer to the additive (A), compensatory (C), and logistic (L) survival functions. The corresponding equilibrium solutions are superimposed on each curve as dotted lines and open circles. The recruitment model used for each graph is indicated (refer to Table 3).

tional forms of the recruitment and survival functions affected the optimal strategy at nonequilibrium values of the state variable. Thus, we wished to compare the optimal decision strategy, $D^{*}\left[N_{t}\right]$, for a number of recruitment/survival model combinations. We used the same 18 parameterizations as shown in Table 3, so that we could superimpose the equilibrium solutions we had already calculated onto the state-dependent solutions.

We used dynamic programming to solve the systems for the optimal decision strategy. This technique requires making the state variable (population size) and the control variable (harvest rate) discrete, but by making the discretization arbitrarily fine, we were able to generate smooth, numerical solutions for $D^{*}\left[N_{t}\right]$. We valued the harvest on a risk-neutral scale, that is, the utility of the harvest was equal to the total number of ducks harvested. We did not discount future harvests, nor did we assign any value to the terminal state of the process. To find the infinite time horizon solution, we used dynamic programming to find iterative solutions backward in time, until the optimal policy converged. Our convergence criterion was a lack of change in the state-dependent optimal policy for four consecutive iterations. In practice, our solutions required between 40 and 50 iterations to converge. To calculate the optimal strategy, we used SDP (Lubow 1995), a flexible computer program for solving both deterministic and stochastic dynamic programming problems.

\section{Results}

We found substantial differences in the pattern of the optimal strategy among the different recruitment and survival models (Fig. 5). The optimal decision strategy under the additive survival model is to not harvest if the population size is quite small, but to harvest at increasing levels as the population size increases, with one exception: the relationship between optimal harvest rate and population size need not be monotonic increasing. Under the linear model the optimal harvest rate will actually begin to decrease at high population sizes (Fig. 5B) as a consequence of “overcompensation" (Quinn and Deriso 1999). As population size increases, the recruitment rate decreases faster than the population size increases, so 
the total recruitment declines. Thus, there is less "surplus" to harvest. (Note that the discontinuity in the slope of this graph is a result of the piecewise definition of the linear recruitment function. See Eq. 5). The optimal harvest strategies for the density-dependent logistic survival function show the same pattern as for the additive survival model, with shifts in the curve reflecting the effect of the survival function. But note that the order of these curves (A and $\mathrm{L}$ on the graphs in Fig. 5) can vary. In recruitment model 2 (Fig. 5B), the harvest rate under the logistic survival model is always higher than under the additive survival model, in contrast to the other five examples. In this case, note that the optimal equilibrium population sizes are fairly close together, in other words, the goal is to hold the population at roughly the same point under the two models. Because the logistic model has the property of harvest compensation, a higher harvest rate is allowable. So, is it counterintuitive that in the other five examples, the optimal harvest rate under the additive model is always higher than under the logistic model? In the other cases, the optimal equilibrium population size is much lower under the additive model than under the logistic model. Thus, the higher harvest rates under the additive model reflect the need to move the population size to a lower equilibrium point. The compensatory survival model showed much different behavior than the other two. At low population size, the optimal strategy was to harvest at the "threshold," that is, to take the maximum harvest that would still not have an impact on the population (the "free" harvest). For the linear and exponential recruitment functions, the optimal harvest rate under the compensatory model does increase above the threshold for high population sizes. But for the hyperbolic recruitment function, the optimal strategy under the compensatory survival model is to harvest at the threshold rate, regardless of the population size!

By superimposing the equilibrium solutions on the graphs of the optimal state-dependent decision strategies, we found that (1) the equilibrium solution cannot be found simply by looking at the graph of the optimal strategy; and (2) the spacing of the optimal strategies for different survival models depends on the differences between the optimal equilibrium population size under these models. As noted above, while the optimal equilibrium population sizes for the three survival models were always observed to be in the same order (additive $<$ logistic $<$ compensatory), the optimal harvest rates that led to these equilibria could take many different orders.

\section{The Effect of Stochasticity}

The population model discussed above is deterministic and thus the optimal decision strategy can be found using dynamic programming. Do the results change when the population model contains stochastic com- ponents? To answer this question, we created a stochastic version of the population model, and solved for the optimal decision policy using stochastic dynamic programming.

The stochastic version of the population model made the recruitment and the survival stochastic. The recruitment, $R\left[N_{t}\right]$, was treated as a normally distributed random variable with mean given by Eqs. 5, 6 , or 7 and a coefficient of variation denoted by $\mathrm{CV}_{\mathrm{R}}$. Survival, $F\left[P_{t}\right]$, was treated as a normally distributed random variable with mean given by Eqs. 12, 13, or 15 and a standard deviation of $\mathrm{SD}_{\mathrm{S}}$ (for the compensatory survival model, the total annual survival, i.e., $(1-h) F\left[P_{t}\right]$, rather than just $F\left[P_{t}\right]$, was treated in this manner). We used a rough discretization of the normal distribution to generate random values, since the method of stochastic dynamic programming requires discrete outcomes in addition to discrete state and decision variables. We used the $0.1,0.3,0.5,0.7$, and 0.9 quantiles of the standard normal distribution as values of a discrete random variable, each chosen with probability 0.2 . These were then multiplied by the appropriate standard deviation (as calculated from the coefficient of variation and the mean) and added to the appropriate mean. When necessary, all random distributions were truncated to the appropriate biological interval, that is, $(0, \infty)$ for $R[\cdot]$ and $(0,1)$ for $F[\cdot]$. We considered all nine combinations of three values for $\mathrm{CV}_{\mathrm{R}}(0.0,0.15$, and 0.50$)$ and three values for $\mathrm{SD}_{\mathrm{S}}(0.0,0.1$, and 0.25$)$. We used SDP (Lubow 1995) to solve for the optimal decision strategy for all three survival models, using the exponential recruitment model (model 3; see Table 3). All other details of the method were as described above for the deterministic case, except we used a coarser discretization of the state variable, in order to reduce computation time.

We observed no effect of stochasticity in the recruitment function on the optimal decision strategy (Fig. 6). In some simulations not included here, we found that high stochastic variation in the survival function (e.g., $\mathrm{SD}_{\mathrm{S}}=0.5$ ) appeared to shift the optimal decision curves, but closer examination revealed that this shift was exactly due to the effect of truncating the probability distributions. The truncation caused a shift in the mean of the survival rate distribution. When we reran the solution using a distribution with the altered mean, we calculated the same optimal policy. Thus, the results of this study show no effect of stochasticity on the optimal decision strategy, for the population model and limited number of scenarios considered.

\section{DISCUSSION}

The choice of functional form in population models is not trivial, as it can have a substantial impact on the optimal harvest rate, the equilibrium population size, and the state-dependent optimal policy. This conclu- 

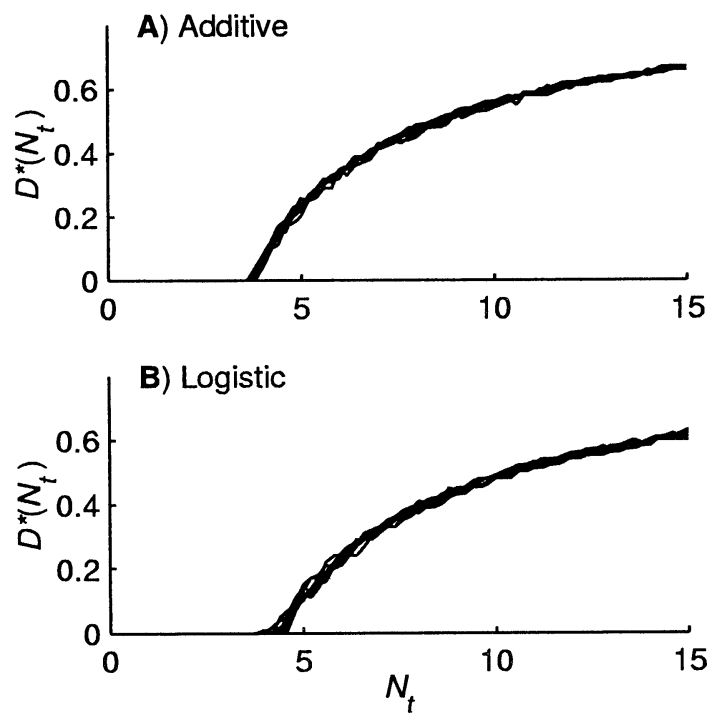

FIG. 6. State-dependent optimal harvest rate as a function of current population size, stochastic results. Exponential recruitment, model 3 (Table 3), was combined with each of the three survival functions (compare to Fig. 5C). Nine curves are shown on each graph, one for each of the combinations of the three levels of variance in the reproductive function $\left(\mathrm{CV}_{\mathrm{R}}=0.0,0.15\right.$, and 0.5$)$ with the three levels of variance in the survival function $\left(\mathrm{SD}_{\mathrm{S}}=0.0,0.1,0.25\right)$. (A) Additive survival model; (B) logistic survival model. The optimal policies under the compensatory survival model were exactly the same for the nine stochastic cases as for the deterministic case (graph not shown).

sion may seem obvious, and indeed, it has been described or at least implied by others (e.g., Ricker 1954, Beverton and Holt 1957, Holling 1965, Paulik 1973, Walters 1986) but it bears reinforcement, particularly for the applied ecologist. One of the important implications is that multiple forms may fit a given data set equally well (i.e., be statistically indistinguishable) but may result in very different optimal solutions because of differences that lie outside the range of the observed, or even the anticipated, data. Thus, an applied ecologist needs to think not only about the ecological integrity and statistical fit of a model, but also needs to consider the resultant properties of the optimal solution. Pascual et al. (1997) made this point well with regard to viability analyses. We have shown that the importance is retained in dynamic control models.

When there are multiple competing models for system dynamics, and these models produce large differences in the optimal control policies, management of the system can be greatly enhanced by resolution of the structural uncertainty. This is the basic tenet of adaptive management (Walters 1986). Alternative models that differ in functional form may be indistinguishable based on current knowledge but may differ greatly in the resultant optimal policies. Thus, this sort of structural uncertainty may warrant active probing-allowing the system to move outside the realm of past experience in order to distinguish be- tween the alternative models. Development of alternative models with a confidence ellipsoid approach (that is, by choosing several sets of parameter estimates that are on extremes of the multidimensional profile likelihood) focuses on identifying uncertainty within the range of the data, while assuming a single functional form for the system dynamics. In some settings, such uncertainty may not be as important as uncertainty about functional form.

We were surprised to find such a variety of patterns in the optimal solutions, even with such a simple population model, and have realized that it is not easy to develop an intuitive sense of what such solutions should look like. One of the key insights we gained from this work is that the state-dependent optimal policies need to be understood in reference to the corresponding optimal equilibrium points. For instance, we observed that state-dependent optimal harvest rates for an additive survival model are often higher than those for a density-dependent survival model (a result we initially thought was counterintuitive) because the equilibrium population size sought under the additive model is much lower than that for the density-dependent model (e.g., Fig. 5A, C-F). When the optimal equilibrium population sizes are close together for the additive and logistic models, the statedependent optimal harvest rates are higher for the logistic model (Fig. 5B), because increased harvest in the logistic model is compensated by an increase in the postharvest survival rate. But there are also properties of the state-dependent optimal policy that are not evident from the equilibrium solution. Compare, for instance, the additive and compensatory survival models for the linear recruitment model (Fig. 5A) and the hyperbolic recruitment model (Fig. 5E): in both cases the optimal equilibrium population size for the compensatory model is $K=10$, and that for the additive model is substantially lower; the optimal harvest rate at equilibrium is also lower for the additive than the compensatory model in both cases; and the optimal policy for the additive survival model looks about the same under both recruitment models. But under the hyperbolic recruitment model (Fig. 5E), the optimal policy under the compensatory hypothesis is to harvest at the threshold rate, regardless of population size. However, under the linear recruitment model, there is a population size above which the optimal harvest rate rises above the threshold. The reason for this difference is that under the hyperbolic recruitment model, there is still significant positive recruitment at high population size. Rather than move the population back to the desired equilibrium point quickly (through higher harvest), it is more advantageous to let it drop back to that point on its own, while taking all the "free" harvest. The point of this labored contrast is that the nature of the path to the equilibrium, which determines the state-dependent 
optimal policy, is strongly affected by the functional form of the system dynamics.

The primary results and conclusions of this paper are derived from deterministic models, and while the results also held under some stochastic conditions, congruence of deterministic and stochastic results is not a general conclusion. In the model we used there was one absorbing state $\left(N_{t}=0\right)$ that would have a negative impact on the long-term objective, but there was no basin of attraction for this state. We included environmental, but not demographic, stochasticity in the model, so, unless harvest was set to $h_{t}=1$, there was zero probability of ever getting to $N_{t}=0$. One way that optimal control results will differ between deterministic and stochastic versions of the same population model is that, in the stochastic situation, the optimal decision policy will avoid getting near a basin of attraction to an absorbing state. Thus, if we had included an Allee effect in the model, increased variance in recruitment or survival would result in a more conservative harvest policy, in order to avoid accidentally ending up in the basin of attraction to $N_{t}=0$ (Dennis 1989, Alvarez 1998, Stephens and Sutherland 1999). Another aspect of our study that minimized the effect of stochasticity was that we were looking at equilibrium solutions in a density-dependent model. If we had been focusing on a situation where long-term projections of an increasing or decreasing population were of interest (such as in population invasion or viability analyses), the effects of stochasticity may have been significant. Further, note that in our simulations we used symmetric distributions to capture environmental stochasticity. Skewed distributions, which are often biologically relevant, might affect the optimal policy under stochastic, as compared to deterministic, conditions, since they are known to have an impact on growth rates in stochastic models (Slade and Levenson 1984). The issue of how to model the stochastic elements (for instance, whether to use a normal or beta distribution for survival rate) is in some sense an issue about functional form. In that manner, it may well fit under the general concern about process uncertainty that we have grappled with in this paper. Certainly, further investigation of the differences in the optimal decision policy under deterministic and stochastic models is warranted.

In addition to the general conclusions discussed above, there are a number of conclusions that are more specific to the general framework we considered (i.e., Eq. 1). Beginning with the equilibrium solutions, (1) under the additive survival model and the exponential or hyperbolic recruitment models, the optimal equilibrium population size is always smaller than $K / 2$, the optimal equilibrium point for the linear recruitment model (compare the solutions in Table 1). Both of these recruitment functions are convex over their entire range $\left(R^{\prime \prime}[N]>0\right)$. A func- tion that was concave would have an optimal equilibrium population size greater than $K / 2$, as in the generalized logistic equation (Wade 1998). (2) An important property of the recruitment function appears to be the characteristics of the left tail, i.e., how steep the curve is near $N=0$. When the curve can become nearly vertical (as in the hyperbolic model), the optimal harvest rate at equilibrium under the additive model can be higher than that for the compensatory and density-dependent models. While this is not necessarily unsupportable, it does raise the ecological question of how fast recruitment can increase at low densities. (3) For any given parameterization of a recruitment model, the optimal equilibrium population size and the optimal annual harvest will be greater for the logistic survival model than for the additive survival model. The reason is that the logistic model provides a mechanism for harvest compensation, so while there isn't any "free harvest," the harvest comes at a reduced price, in that increased harvest is compensated for by increased postharvest survival.

In regard to the state-dependent solutions, (1) an important property of the recruitment function appears to be the area under the curve. If this is infinite (as in the hyperbolic model), the state-dependent harvest rate under the compensatory model never rises about the threshold. If the area under the curve is finite (as in the exponential model), the state-dependent harvest rate can rise about the threshold. In the latter case, the decrease in recruitment at high densities is fast enough to warrant additional harvest, in order to move toward the optimal equilibrium point more quickly. The exponential model considered here is related to the Ricker stock-recruitment curve (Ricker 1954) and the hyperbolic model is related to the Beverton-Holt stock-recruitment curve (Beverton and Holt 1957), so the question about the nature of recruitment at high densities is not new. (2) As noted above, the state-dependent optimal harvest rates under the additive survival model are often greater than under the logistic survival model, a consequence of the different optimal equilibrium population sizes. But when the equilibrium population sizes are close, the optimal harvest rates under the logistic model are higher, as a consequence of harvest compensation.

The most divergent results we saw were connected with the compensatory survival model. This model has a long history in the duck literature, and is used as an alternative model in current practice with mallards (Johnson et al. 1997), but may not be a satisfactory ecological hypothesis. Note that, strictly speaking, this is not a density-dependent survival model-the annual survival rate depends on the harvest rate, not the resulting population density. Thus, two populations with the same postharvest density might have quite different average survival rates if the harvest rates were very different. If the compensatory model is meant to be a 
phenomenological characterization of a density-dependent situation, it will only make good predictions when the harvest rate is strongly correlated with the population density. If the compensatory model is used as an alternative to the additive model in an adaptive management setting, but the true survival mechanism is density-dependent, then support for one alternative model or the other (as measured by the Bayesian posterior probabilities) will not increase monotonically but will fluctuate depending on whether the population density is high or low. As an ecological hypothesis, the compensatory model implies that the survival rate in the population is not density dependent. Rather, animals that would have died anyway are preferentially harvested, that is, the harvest is not a random sample of the population (Burnham and Nichols 1985, Johnson et al. 1986). While that is a tenable hypothesis (more so in the context of predation than harvest, where the weak and ill may be preferentially killed), we are more inclined to seek a mechanistic density-dependent hypothesis for fluctuations in annual survival of ducks (Johnson et al. 1993).

Three general caveats should be mentioned about the results in this paper. First, the optimal decision policy depends critically on the objective function. We have only looked at one objective function, long-term maximization of harvest, and we expect that different objective functions will have a much more profound impact on the optimal policies than the functional form of the population model. For many objective functions, we expect that the importance of functional form would be preserved. For others, functional form may be a secondary consideration. For instance, when the objective function involves maintaining a population size in a narrow range, functional forms that are similar in that range may be practically equivalent, and differences outside that range may be irrelevant. Or, if the objective function is to maximize harvest, but with heavy discounting, the short-term dynamics will be more important, so the optimal policies may not differ greatly between models with different functional forms. Second, the results of this paper are not comprehensive. We have only looked at a small set of possible functional forms for recruitment and survival. Other functional forms and other model structures will likely produce results not anticipated here. Some considerations that might be important include: existence of an Allee effect; whether the survival depends on prebreeding or postharvest density (i.e., time lags in the model structure); demographic stochasticity; the influence of additional stochastic environmental factors on reproduction and survival; and individual heterogeneity in vital rates. Third, we should also understand the notion of functional form more broadly. In this sense, different formulations of age, sex, or size structure in the population model constitute process uncertainty. For instance, in the model we used (Eq. 1), we assumed that postharvest survival was the same for adults and young. This assumption, and whether the two cohorts respond differently to density, could have a strong effect on the solutions of the optimal control problem.

This work reiterates the lesson that model development should be based on sound mechanistic ecological principles rather than statistical fit. The functional forms used to express population processes can have a profound effect on optimal decision policies, even when they do not differ in the range of past experience. When developing alternative models to express uncertainty about system dynamics, it may be wise to focus attention on what the different models predict in the extremes of the state-space. The use of adaptive management to resolve uncertainty about functional form is an appropriate way to address these concerns in the applied setting.

\section{ACKNOWLEDGMENTS}

This work was motivated by conversations with Susan E. Sheaffer and James D. Nichols, and benefited greatly from their comments and critiques. We would also like to thank Michael J. Conroy, William L. Kendall, and two anonymous reviewers for their helpful advice.

\section{LiTERATURE Cited}

Alvarez, L. H. R. 1998. Optimal harvesting under stochastic fluctuations and critical depensation. Mathematical Biosciences 152:63-85.

Anderson, D. R., and K. P. Burnham. 1976. Population ecology of the mallard. VI. The effect of exploitation on survival. Resource Publication 128. United States Fish and Wildlife Service, Washington, D.C., USA.

Beverton, R. J. H., and S. J. Holt. 1957. On the dynamics of exploited fish populations. Fishery Investigations Series 2(19). Ministry of Agriculture, Fisheries, and Food, London, UK.

Boyce, M. S., A. R. E. Sinclair, and G. C. White. 1999. Seasonal compensation of predation and harvesting. Oikos 87:419-426.

Burnham, K. P., and J. D. Nichols. 1985. On condition bias and band-recovery data from large-scale waterfowl banding programs. Wildlife Society Bulletin 13:345-349.

Clark, C. W. 1987. The lazy, adaptable lions: a Markovian model of group foraging. Animal Behavior 35:361-368.

Clark, C. W., and D. A. Levy. 1988. Diel vertical migrations by juvenile sockeye salmon and the antipredation window. American Naturalist 131:271-290.

Clark, C. W., and M. Mangel. 2000. Dynamic state variable models in ecology: methods and applications. Oxford University Press, New York, New York, USA.

Cohen, Y. 1987. A review of harvest theory and applications of optimal control theory in fisheries management. Canadian Journal of Fisheries and Aquatic Science 44:75-83.

Corless, R. M., G. H. Gonnet, D. E. G. Hare, D. J. Jeffrey, and D. E. Knuth. 1996. On the Lambert $W$ function. Advances in Computational Mathematics 5:329-359.

Dennis, B. 1989. Allee effects: population growth, critical density, and the chance of extinction. Natural Resource Modeling 3:481-538.

Eadie, J. M., F. P. Kehoe, and T. D. Nudds. 1988. Pre-hatch and post-hatch brood amalgamation in North American Anatidae: a review of hypotheses. Canadian Journal of Zoology 66:1709-1721.

Holling, C. S. 1965. The functional response of predators to prey density and its role in mimicry and population reg- 
ulation. Memoirs of the Entomological Society of Canada 45:5-60.

Houston, A., C. Clark, J. McNamara, and M. Mangel. 1988. Dynamic models in behavioural and evolutionary ecology. Nature 332:29-34.

Houston, A. I., and J. M. McNamara. 1986. Singing to attract a mate-a stochastic dynamic game. Journal of Theoretical Biology 129:171-180.

Johnson, D. H., K. P. Burnham, and J. D. Nichols. 1986. The role of heterogeneity in animal population dynamics. Proceedings of the Thirteenth International Biometrics Conference. Biometric Society, University of Washington, Seattle, Washington, USA.

Johnson, F. A., C. T. Moore, W. L. Kendall, J. A. Dubovsky, D. F. Caithamer, J. R. Kelley, and B. K. Williams. 1997. Uncertainty and the management of mallard harvests. Journal of Wildlife Management 61:202-216.

Johnson, F. A., B. K. Williams, J. D. Nichols, J. E. Hines, W. L. Kendall, G. W. Smith, and D. F. Caithamer. 1993. Developing an adaptive management strategy for harvesting waterfowl in North America. Transactions of the North American Wildlife and Natural Resources Conference 58: $565-583$.

Lancia, R. A., C. E. Braun, M. W. Collopy, R. D. Dueser, J. G. Kie, C. J. Martinka, J. D. Nichols, T. D. Nudds, W. R. Porath, and N. G. Tilghman. 1996. ARM! for the future: adaptive resource management in the wildlife profession. Wildlife Society Bulletin 24:436-442.

Lubow, B. C. 1995. SDP: generalized software for solving stochastic dynamic optimization problems. Wildlife Society Bulletin 23:738-742.

MacArthur, R. H. 1960. On the relation between reproductive value and optimal predation. Proceeding of the National Academy of Sciences USA 46:143-145.

Mangel, M., and C. W. Clark. 1986. Towards a unified foraging theory. Ecology 67:1127-1138.

Mangel, M., and C. W. Clark. 1988. Dynamic modeling in behavioral ecology. Princeton University Press, Princeton, New Jersey, USA.

McCullagh, P., and J. A. Nelder. 1989. Generalized linear models. Chapman and Hall, London, UK.

McNamara, J. M., R. H. Mace, and A. I. Houston. 1987. Optimal daily routines of singing and foraging in a bird singing to attract a mate. Behavioral Ecology and Sociobiology 20:399-405.

Nichols, J. D. 1991. Responses of North American duck populations to exploitation. Pages $498-525$ in C. M. Perrins, J.-D. Lebreton, and G. J. M. Hirons, editors. Bird populations studies: relevance to conservation and management. Oxford University Press, Oxford, UK.

Nichols, J. D., M. J. Conroy, D. R. Anderson, and K. P. Burnham. 1984. Compensatory mortality in waterfowl populations: a review of the evidence and implications for research and management. Transactions of the North American Wildlife and Natural Resources Conference 49:535554.

Nichols, J. D., and F. A. Johnson. 1996. The management of hunting of Anatidae. Gibier Faune Sauvage, Game and Wildlife 13:977-989.
Nichols, J. D., F. A. Johnson, and B. K. Williams. 1995. Managing North American waterfowl in the face of uncertainty. Annual Review of Ecology and Systematics 26: 177-199.

Pascual, M. A., P. Kareiva, and R. Hilborn. 1997. The influence of model structure on conclusions about the viability and harvesting of Serengeti wildebeest. Conservation Biology 11:966-976.

Paulik, G. J. 1973. Studies of the possible form of the stockrecruitment curve. Pages 302-315 in B. B. Parrish, editor. Fish stocks and recruitment. Rapports et Procès-Verbaux des Réunions 164. Conseil International pour 1'Exploration de la Mer, Copenhagen, Denmark.

Puterman, M. L. 1994. Markov decision processes: discrete stochastic dynamic programming. John Wiley and Sons, New York, New York, USA.

Quinn, T. J., II, and R. B. Deriso. 1999. Quantitative fish dynamics. Oxford University Press, New York, New York, USA.

Ricker, W. E. 1954. Stock and recruitment. Journal of the Fisheries Research Board of Canada 11:559-623.

Runge, M. C. 1999. Design and analysis of a model for adaptive harvest management of beaver (Castor canadensis). Dissertation. Cornell University. Ithaca, New York, USA.

Schaffer, W. M. 1983. The application of optimal control theory to the general life history problem. American Naturalist 121:418-431.

Sheaffer, S. E., M. C. Runge, and R. A. Malecki. 1999. Models for adaptive harvest management of northern pintails. Report to the Pacific Flyway Waterfowl Council Study Committee. New York Cooperative Fish and Wildlife Research Unit, Ithaca, New York, USA.

Slade, N. A., and H. Levenson. 1984. The effect of skewed distributions of vital statistics on growth of age-structured populations. Theoretical Population Biology 26:361-366.

Slobodkin, L. B. 1968. How to be a predator. American Zoologist 8:43-51.

Stephens, P. A., and W. J. Sutherland. 1999. Consequences of the Allee effect for behaviour, ecology and conservation. Trends in Ecology and Evolution 14:401-405.

United States Fish and Wildlife Service. 1999. Adaptive harvest management: 1999 duck hunting season. Office of Migratory Bird Management, USFWS, Arlington, Virginia, USA.

Wade, P. R. 1998. Calculating limits to the allowable humancaused mortality of cetaceans and pinnipeds. Marine Mammal Science 14:1-37.

Walters, C. 1986. Adaptive management of renewable resources. Macmillan Publishing Company, New York, New York, USA.

Williams, B. K. 1982. Optimal stochastic control in natural resource management: framework and examples. Ecological Modelling 16:275-297.

Williams, B. K., and J. D. Nichols. 1984. Optimal timing in biological processes. American Naturalist 123:1-19.

Yodzis, P. 1989. Introduction to theoretical ecology. Harper and Row, New York, New York, USA.

\section{APPENDIX}

In this appendix, we show a sample calculation of the equilibrium solution, using the hyperbolic recruitment model and the additive survival model. The recruitment is given by Eq. 7 as

$$
R\left[N_{t}\right]=\frac{b}{a+N_{t}}
$$

and the postharvest survival is given by Eq. 12 as

$$
F\left[P_{t}\right]=\phi .
$$

To find the equilibrium population size in the absence of harvest $(K)$, we use Eq. 4, which implies that

$$
\frac{1-\phi}{\phi}=R[K]=\frac{b}{a+K}
$$

the solution to which is 


$$
K=\frac{\phi b}{1-\phi}-a .
$$

To find the equilibrium population size at a constant harvest rate, we begin with Eq. 20, substitute in the appropriate expressions for $R[\cdot]$ and $F[\cdot]$, then solve for $N_{\text {eq }}(h)$. Since we are using the additive survival model in this example, we can begin with Eq. 24, and substitute in Eq. A1. This gives

$$
\frac{b}{a+N_{\mathrm{eq}}}=\frac{1-\phi(1-h)}{\phi(1-h)}
$$

the solution for which is

$$
N_{\mathrm{eq}}(h)=\frac{\phi b(1-h)}{1-\phi(1-h)}-a .
$$

The annual harvest as a function of harvest rate can be calculated by inserting Eq. A6 into Eq. 25. Thus,

$$
H(h)=\left[\frac{\phi b(1-h)}{1-\phi(1-h)}-a\right]\left(\frac{h}{\phi(1-h)}\right) .
$$

To find the harvest rate that achieves the maximum annual harvest, we need to take the derivative of Eq. A7 with respect to $h$, set it equal to 0 , and solve for $h$. The derivative of the annual harvest with respect to the harvest rate is

$$
\begin{aligned}
\frac{d H}{d h}= & {\left[\frac{\phi b(1-h)}{1-\phi(1-h)}-a\right]\left(\frac{1}{\phi(1-h)^{2}}\right) } \\
& +\left(\frac{h}{\phi(1-h)}\right)\left[\frac{-\phi b}{(1-\phi(1-h))^{2}}\right] \\
= & \left(\frac{1}{\phi(1-h)}\right)\left\{\frac{\phi b(1-\phi)(1-h)}{(1-\phi(1-h))^{2}}-\frac{a}{1-h}\right\}
\end{aligned}
$$

which is equal to 0 when

$$
\frac{\phi b(1-\phi)(1-h)}{(1-\phi(1-h))^{2}}=\frac{a}{1-h} .
$$

A little algebra reveals this is true when

$$
h^{*}=1-\frac{1}{\sqrt{\frac{\phi b(1-\phi)}{a}+\phi}}
$$

and a lot more calculus and algebra proves that this critical point is in fact a maximum, because the second derivative of $H$ with respect to $h$, evaluated at $h^{*}$, is negative.

We can now substitute this solution for the optimal harvest rate at equilibrium into Eqs. A6 and A7 to find the optimal equilibrium population size and annual harvest. The optimal equilibrium population size is

$$
\begin{aligned}
N^{*} & =\frac{\phi b\left(1-h^{*}\right)}{1-\phi\left(1-h^{*}\right)}-a=\frac{b}{\left[\frac{1}{\phi\left(1-h^{*}\right)}-1\right]}-a \\
& =\frac{\phi b}{\sqrt{\frac{\phi b(1-\phi)}{a}}}-a=\sqrt{\frac{a(\phi b)^{2}}{\phi b(1-\phi)}}-a \\
& =\sqrt{\frac{a b \phi}{1-\phi}}-a=\sqrt{a(K+a)}-a
\end{aligned}
$$

where the last simplification is made by substituting in the expression from Eq. A4. The optimal annual harvest is most easily expressed in terms of the other optimal equilibrium quantities,

$$
H^{*}=N^{*}\left(\frac{h^{*}}{\phi\left(1-h^{*}\right)}\right) .
$$

The other equilibrium solutions shown in Tables 1 and 2 can be derived by following a similar set of steps. 